\title{
Vitamin $D$ deficiency in chronic inflammatory rheumatic diseases: results of the cardiovascular in rheumatology [CARMA] study
}

\author{
Ana Urruticoechea-Arana ${ }^{1 \dagger}$, María A. Martín-Martínez ${ }^{2 \dagger}$, Santos Castañeda ${ }^{3 \dagger}$, Carlos A. Sanchez Piedra ${ }^{2}$, \\ Carlos González-Juanatey ${ }^{4}$, Javier Llorca ${ }^{5}$ Federico Díaz-Gonzalez ${ }^{2,6,7}$, Miguel A. González-Gay ${ }^{8,9 *}$ \\ and on behalf of the CARMA Project Collaborative Group
}

\begin{abstract}
Introduction: The aim was to study the association between 25-hydroxyvitamin $\mathrm{D}(25(\mathrm{OH}) \mathrm{D})$ levels and the clinical characteristics of patients with chronic inflammatory rheumatic diseases (CIRD).

Methods: We studied a cross-section from the baseline visit of the CARMA project (CARdiovascular in rheuMAtology), a 10-year prospective study evaluating the risk of cardiovascular events in rheumatoid arthritis (RA), ankylosing spondylitis (AS) and psoriatic arthritis (PsA) patients, and non-CIRD patients who attended rheumatology outpatient clinics from 67 hospitals in Spain. Non-CIRD group was frequency matched by age with the joint distribution of the three CIRD groups included in the study. $25(\mathrm{OH}) \mathrm{D}$ deficiency was defined if $25(\mathrm{OH}) \mathrm{D}$ vitamin levels were $<20 \mathrm{ng} / \mathrm{ml}$.

Results: 2.234 patients (775 RA, 738 AS and 721 PsA) and 677 non-CIRD subjects were assessed. The median (p25-p75) 25(OH)D levels were: 20.4 (14.4-29.2) ng/ml in RA, 20.9 (13.1-29.0) in AS, 20.0 (14.0-28.8) in PsA, and 24.8 $(18.4-32.6) \mathrm{ng} / \mathrm{ml}$ in non-CIRD patients. We detected 25(OH)D deficiency in $40.5 \%$ RA, $39.7 \%$ AS, $40.9 \%$ PsA and $26.7 \%$ non-CIRD controls $(p<0.001)$. A statistically significant positive association between RA and $25(\mathrm{OH}) \mathrm{D}$ deficiency was found (adjusted (adj.) $\mathrm{OR}=1.46 ; 95 \% \mathrm{Cl}=1.09-1.96) ; \mathrm{p}=0.012$. This positive association did not reach statistical significance for AS (adj. OR 1.23; $95 \% \mathrm{Cl}=0.85-1.80$ ) and PsA (adj. OR 1.32; $95 \% \mathrm{Cl}=0.94-1.84$ ). When the parameters of disease activity, severity or functional impairment were assessed, a marginally significant association between 25(OH)D deficiency and ACPA positivity in RA patients (adj. OR=1.45; $95 \% \mathrm{Cl}=0.99-2.12 ; \mathrm{p}=0.056$ ), and between 25(OH)D deficiency and BASFl in AS patients (adj. OR=1.08; $95 \% \mathrm{Cl}=0.99-1.17$ ); $p=0.07$ ) was also found.
\end{abstract}

Conclusions: Patients with RA show an increased risk of having 25(OH)D deficiency compared to non-CIRD controls.

\section{Introduction}

Vitamin D has raised great interest in recent decades due to its multiple physiological functions, a including significant role in the regulation of the immune system [1-7]. Vitamin D deficiency is an extremely common health problem that affects up to $50 \%$ of the general

\footnotetext{
* Correspondence: miguelaggay@hotmail.com

tEqual contributors

${ }^{8}$ Division of Rheumatology, Hospital Universitario de Canarias, La Laguna, Tenerife, Spain

${ }^{9}$ Division of Rheumatology, Hospital Universitario Marqués de Valdecilla, Santander, and Epidemiology, Genetics and Atherosclerosis Research Group on Systemic Inflammatory Diseases, Rheumatology Division, IDIVAL, Avenida de Valdecilla, s/n, 39008 Santander, Spain

Full list of author information is available at the end of the article
}

population during winter months in the Northern hemisphere [8]. Several studies have pointed out a potential association between vitamin D deficiency and cancer, some chronic infections, cardiovascular mortality and increased risk of some autoimmune diseases [8-13], such as type I diabetes mellitus [10], multiple sclerosis [10], systemic lupus erythematosus (SLE) $[9,11,12]$ and rheumatoid arthritis (RA) [9, 13]. In this regard, some authors have reported an inverse relationship between serum levels of 25-hydroxyvitamin D (25(OH)D) and disease activity or functional impairment in patients with RA or early arthritis [14-21]. In a recent study, vitamin D deficiency was found in $30 \%$ of RA patients [8]. Most studies assessing serum

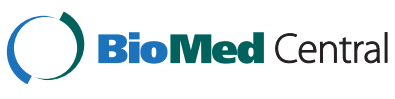

(C) 2015 Urruticoechea-Arana et al. Open Access This article is distributed under the terms of the Creative Commons Attribution 4.0 International License (http://creativecommons.org/licenses/by/4.0/), which permits unrestricted use, distribution, and reproduction in any medium, provided you give appropriate credit to the original author(s) and the source, provide a link to the Creative Commons license, and indicate if changes were made. The Creative Commons Public Domain Dedication waiver (http://creativecommons.org/publicdomain/zero/1.0/) applies to the data made available in this article, unless otherwise stated. 
25(OH)D levels in patients with chronic inflammatory rheumatic diseases (CIRD) were focused on patients with RA [9, 13-15, 17-20]. However, fewer studies have analyzed the presence of $25(\mathrm{OH}) \mathrm{D}$ deficiency in other CIRD such as ankylosing spondylitis (AS) or psoriatic arthritis (PsA) [21-24].

The aim of this study was to assess $25(\mathrm{OH}) \mathrm{D}$ levels in a cohort of Spanish patients with CIRD that included patients with RA, AS, PsA and non-CIRD, who were attending rheumatology outpatient clinics, and to determine the potential relationship between $25(\mathrm{OH}) \mathrm{D}$ levels and clinical characteristics of every disease included in the group of patients with CIRD.

\section{Methods}

\section{Study design}

Cross-sectional analysis from the baseline visit of the project, CARdiovascular in rheuMAtology (CARMA), a 10-year prospective cohort study designed to determine the cardiovascular mortality risk in patients with CIRD compared to a cohort of patients without inflammatory pathological disease [25]. Information on this cohort has recently been reported. The institutional review board of each center approved the study and all patients signed an informed consent agreement.

\section{Patients and controls}

Based on the information from the Spanish Society of Rheumatology (SER), all the Spanish public hospitals (university and general community hospitals) that have rheumatology units were invited to take part in the present study. Finally, 67 (63.2 \%) of the 106 centers agreed to participate in the study. They recruited 2,986 patients who attended rheumatology outpatient clinics from July 2010 to January 2012. Seventy-five patients declined the invitation. Therefore, 2,911 patients over 18 years old were included in the study. They were split into two different cohorts according to CIRD exposition. The CIRD patients consisted of 775 patients diagnosed with RA (1987 American College of Rheumatology (ACR) classification criteria) [26], 738 diagnosed with AS (modified New York criteria) [27] and 721 patients with PsA (Moll and Wright criteria) [28]. The control (non-CIRD) group included 677 patients without inflammatory rheumatic disease. The non-CIRD group was frequency-matched by age with the joint distribution of the three CIRD groups. Therefore, its distribution approximates the joint age distribution of the three CIRD. To see the list of participating centers, we advise readers to see the Acknowledgements section.

\section{Variables and operative definitions}

Both cohorts were evaluated following international protocols including standardized definitions and validated questionnaires. All patients included were continuously and systematically evaluated online and, to verify the quality of the information, an in situ monitoring data assessment was performed in $15 \%$ of patients randomly selected.

The primary endpoint was the presence of $25(\mathrm{OH}) \mathrm{D}$ deficiency defined as 25(OH)D levels below $20 \mathrm{ng} / \mathrm{ml}$. The variable sunshine hours per month and province (geographical area in which the hospital is located) was established considering the hours of sunlight in the period of time between 60 and 90 days prior to the visit of inclusion of each patient. For this purpose we used the information published by the Spanish Meteorological Agency (SMA) [29]. When information on sunshine hours of the month and year was not available, we used the mean value of sunshine hours of the last 5 years in which information on sunshine hours in the same period of time and site was available. The $25(\mathrm{OH}) \mathrm{D}$ analysis was locally performed according to the methodology and reproducibility level of each institution.

Other variables analyzed were: (1) obesity (BMI $\geq 30$, $\mathrm{kg} / \mathrm{m}^{2}$ ) and main physical activity during working hours (low activity: sitting most of the time; moderate activity: standing most of the time and with little movement or effort; intense activity: walking most of the time or performing tasks that require high physical activity); (2) disease characteristics and parameters of disease activity: rheumatoid factor (RF), anti-cyclic citrullinated peptide antibodies (ACPA), HLA-B27 positivity, erythrocyte sedimentation rate (ESR) $\left(\mathrm{mm} / 1^{\text {st }} \mathrm{h}\right)$, ultra-sensitive C-reactive protein (CRP) (mg/l), disease activity score including 28 jointserythrocyte sedimentation rate (DAS28-ESR), health assessment questionnaire (HAQ (0-3)), Bath ankylosing spondylitis disease activity index (BASDAI $(0-10))$ and Bath ankylosing spondylitis functional index (BASFI (0-10)); (3) sociodemographic variables, and (4) other factors: disease severity, duration of the disease and therapies administered including calcium and vitamin D supplementation.

\section{Statistical analysis}

Numerical variables with a normal distribution were expressed as mean and standard deviation. Variables not normally distributed were shown as median and interquartile range (IQR, percentile (p)25 - p75). Absolute and relative frequencies of qualitative variables were calculated. We performed analysis of the main demographic and clinical variables stratified by type of disease. Stratified analysis of $25(\mathrm{OH}) \mathrm{D}$ deficiency $(<20 \mathrm{ng} / \mathrm{ml})$ was performed for each group of patients according to sociodemographic characteristics and clinical factors, using the Student $t$ test or the Mann-Whitney $U$ test. Qualitative variables were assessed using the Chi-square test, Yates correction, or Fisher test using $2 \times 2$ tables.

To study the association between $25(\mathrm{OH}) \mathrm{D}$ deficiency and CIRD, logistic regression models were constructed 
by calculating the odds ratios (OR) with $95 \% \mathrm{CI}$ and adjusting for potential confounding factors. In this regard, an adjusted model for sunshine hours was performed. It was carried out considering for this purpose the period of time between 60 and 90 days prior to the baseline visit blood test, which included assessment of $25(\mathrm{OH}) \mathrm{D}$ levels. To reduce variability in the methods of measurement of 25(OH)D from each participating hospital, the mixed logistic regression models were constructed with robust variance estimators using the hospital as cluster variable estimation. The same procedure was carried out to identify specific features of each disease. The selection of independent variables in the multivariate models was based on clinical judgment and those with a $p$-value $<0.20$ in the bivariate analysis. In all models of logistic regression, the independent variables were adjusted for the other variables in the model.

Data management and statistical analysis were centralized at the Research Unit of the SER following a pre-established analysis plan. All the analyses were performed using the SPSS 21.0 statistical program. Statistical significance was assumed at $p<0.05$.

\section{Results}

\section{Sociodemographic and clinical characteristics}

Demographic and clinical characteristics of patients included in this study are summarized in Table 1 . There was a predominance of women in the group with RA, whereas most AS patients were men. Sex distribution was similar in the group with PsA. The mean age in patients with RA was higher than in patients with other CIRD. Although patients with AS were younger, the duration of disease was longer than in the other groups. The frequency of obesity was higher among PsA patients, whereas it was lower among controls, despite being the group that included more sedentary individuals $(p<0.001)$. Smoking history was more commonly observed in those with AS.

It is worth noting that a great majority of patients with CIRD included in the study had low disease activity at time of recruitment. In this regard, CRP and ESR levels in RA, AS and PsA patients were remarkably low at the time of inclusion in the study, as well as the functionality scores (HAQ and BASFI, respectively).

Individuals without CIRD (controls) had the following rheumatic diseases: osteoarthritis (30\%), osteoporosis (15.2\%), soft tissue disorders $(18.8 \%)$ and other noninflammatory diseases (36 \%). The distribution at recruitment of individuals with CIRD per hospital was uniform throughout the year 2011, whereas the recruitment of patients without CIRD occurred mainly between October and November 2011 (Additional file 1: Table S1).

\section{5(OH)D levels and CIRD}

Patients with CIRD had lower 25(OH)D levels than those from the non-CIRD controls (Table 1). The median (p25 - p75) 25(OH)D levels were: $20.4(14.4-29.2)] \mathrm{ng} / \mathrm{ml}$ in RA, $20.9(13.1-29.00 \mathrm{ng} / \mathrm{ml}$ in AS, $20.0(14.0-28.8)$ $\mathrm{ng} / \mathrm{ml}$ in PsA and $24.8(18.4-32.6) \mathrm{ng} / \mathrm{ml}$ in non-CIRD individuals. Globally, 25(OH)D deficiency was detected in $40.5 \%$ of patients with RA, $39.7 \%$ of patients with AS, $40.9 \%$ of patients with PsA and $26.7 \%$ of individuals with non-CIRD $(p<0.001)$. The median of sunshine hours in the group of non-CIRD controls was higher than in the three groups of patients with CIRD (Table 2).

Among the variables related to activity and severity of inflammatory diseases, ACPA-positive RA patients had a higher frequency of $25(\mathrm{OH}) \mathrm{D}$ deficiency $(66.9 \%)$. It was also the case for AS, with higher values of BASDAI and BASFI in $25(\mathrm{OH}) \mathrm{D}$-deficient patients $(p<0.05$ in both cases) (Table 2).

\section{Multivariate analysis}

Multivariate analysis (Table 3 ) disclosed a positive association with $25(\mathrm{OH}) \mathrm{D}$ deficiency in the patients with CIRD when compared with the non-CIRD subjects. This association with $25(\mathrm{OH}) \mathrm{D}$ deficiency was statistically significant in the group of patients with RA (adjusted (adj.) $\mathrm{OR}=1.46 ; 95 \% \mathrm{CI}=1.09,1.96) ; p=0.012$. However, the positive association was not statistically significant for AS (adj. OR $=1.23 ; 95 \% \mathrm{CI}=0.85,1.80$ ) and PsA (adj. $\mathrm{OR}=1.32 ; 95 \% \mathrm{CI}=0.94,1.84)$. Women with $\mathrm{RA}$ had significantly higher risk of $25(\mathrm{OH}) \mathrm{D}$ deficiency than men $(p<0.01)$. Likewise, obese RA and PsA patients $(\mathrm{BMI} \geq 30)$ had higher risk of 25(OH)D deficiency

When the parameters of disease activity, severity or functional impairment were assessed, a marginally significant association between 25(OH)D deficiency and ACPApositivity in RA patients (adj. $\mathrm{OR}=1.45 ; 95 \% \mathrm{CI}=0.99$, 2.12; $p=0.056)$, and between $25(\mathrm{OH}) \mathrm{D}$ deficiency and BASFI in AS patients (adj. OR = 1.08; $95 \% \mathrm{CI}=0.99,1.17$; $\mathrm{p}=0.07$ ) was also found (Table 3 ).

\section{Discussion}

Our results show that Spanish patients with RA attending rheumatology outpatient clinics have $25(\mathrm{OH}) \mathrm{D}$ deficiency. This baseline result is from a cohort of patients that has been followed prospectively to determine the cardiovascular outcome. To establish comparisons, we also assessed baseline 25(OH)D levels in non-CIRD controls attending the same rheumatology outpatient clinics [25].

Vitamin D plays an important role in the immune regulation [2]. Vitamin D deficiency has been observed in some autoimmune diseases, in particular in SLE $[11,12,30]$ and RA $[8,20]$. However, information related to undifferentiated spondyloarthropathies and AS is limited [21, 22, 31]. It is also the case for PsA [24].

In our series, the frequency of $25(\mathrm{OH}) \mathrm{D}$ deficiency (level $<20 \mathrm{ng} / \mathrm{ml}$ ) was higher in patients with RA than in the individuals from the non-CIRD control group. The 
Table 1 Sociodemographic characteristics, 25(OH)D levels and clinical characteristics of the population included in the study

\begin{tabular}{|c|c|c|c|c|}
\hline & $\begin{array}{l}\text { Rheumatoid arthritis } \\
(\mathrm{n}=775)\end{array}$ & $\begin{array}{l}\text { Ankylosing spondylitis } \\
(n=738)\end{array}$ & $\begin{array}{l}\text { Psoriatic arthritis } \\
(n=721)\end{array}$ & $\begin{array}{l}\text { Controls } \\
(n=677)\end{array}$ \\
\hline Age at inclusion, years, mean (SD) & $57.1(12.3)$ & $48.1(11.7)$ & $51.8(12.0)$ & $54.0(12.4)$ \\
\hline Age at the beginning of disease, years, mean (SD) & $45.8(13.4)$ & $29.7(11.8)$ & $39.5(13.3)$ & $48.5(12.4)$ \\
\hline Female sex, n (\%) & $581(75.0)$ & $200(27.1)$ & $327(45.4)$ & $437(64.5)$ \\
\hline \multicolumn{5}{|l|}{ Educational level, n (\%) } \\
\hline Elementary & $467(60.9)$ & $318(43.3)$ & $331(46.3)$ & $229(34.1)$ \\
\hline University /secondary & $300(39.1)$ & $416(56.7)$ & $383(53.7)$ & $443(65.9)$ \\
\hline Caucasian Race, n (\%) & $747(96.6)$ & $723(98.0)$ & $712(98.9)$ & $668(98.7)$ \\
\hline Others & $26(3.4)$ & $15(2.0)$ & $8(1.1)$ & $9(1.3)$ \\
\hline \multicolumn{5}{|l|}{ Main activity, $\mathrm{n}(\%)$} \\
\hline Sedentary & $236(35.0)$ & $263(39.3)$ & $253(38.9)$ & $291(46.3)$ \\
\hline Moderate & $290(43.0)$ & $238(35.5)$ & $241(37.1)$ & $207(32.9)$ \\
\hline Active with displacement & $148(22.0)$ & $169(25.2)$ & $156(24.0)$ & $131(20.8)$ \\
\hline BMl, kg/m2, mean (SD) & $26.9(4.8)$ & $27.4(4.4)$ & $28.2(4.7)$ & $26.7(4.4)$ \\
\hline Obesity (BMI 30), n (\%) & $180(23.2)$ & $186(25.2)$ & $209(29.1)$ & $147(21.8)$ \\
\hline \multicolumn{5}{|l|}{ Smoking status, n (\%) } \\
\hline Current smokers & $189(24.4)$ & $254(34.4)$ & $157(21.8)$ & $143(21.2)$ \\
\hline Past smokers & $202(26.1)$ & $240(32.5)$ & $227(31.5)$ & $176(26.0)$ \\
\hline Never smokers & $384(49.5)$ & $244(33.1)$ & $337(46.7)$ & $357(52.8)$ \\
\hline \multicolumn{5}{|l|}{$25(\mathrm{OH}) \mathrm{D}$} \\
\hline 25(OH)D (ng/ml), median (p25 - p75) & $20.4(14.4-29.2)$ & $20.9(13.1-29.0)$ & $20.0(14.0-28.8)$ & $24.8(18.4-32.6)$ \\
\hline 25(OH)D deficiency, n (\%) & $314(40.5)$ & $293(39.7)$ & $295(40.9)$ & $181(26.7)$ \\
\hline Sunshine hours/month*, median (p25 - p75) & $162(122-219)$ & $165(136-233)$ & $178(128-235)$ & $301(202-345)$ \\
\hline Disease duration, years, median (p25 - p75) & $8.0(3.0-14.0)$ & $15.0(8.0-26.0)$ & $9.0(4.0-16.0)$ & $2.0(0.0-6.0)$ \\
\hline RF-positive, n (\%) & $528(68.1)$ & - & - & - \\
\hline ACPA-positive, n (\%) & $482(62.2)$ & - & - & - \\
\hline DAS28-ESR, mean (SD) & $3.2(1.2)$ & - & $3.0(1.3)$ & - \\
\hline HAQ (0-3), median (p25 - p75) & $0.5(0.1-1.1)$ & - & $0.4(0.0-0.9)$ & - \\
\hline ESR (mm/h), median (p25 - p75) & $17.0(9.0-29.0)$ & $10.0(6.0-21.0)$ & $12.0(6.0-21.0)$ & $10.0(5.0-18)$ \\
\hline CRP (mg/l), median (p25 - p75) & $3.1(1.2-8.0)$ & $3.6(1.6-8.9)$ & $2.9(1.4-6.1)$ & $1.9(1.3-3.3)$ \\
\hline BASDAI (0-10), median (p25 - p75) & - & $3.5(1.7-5.3)$ & - & - \\
\hline BASFI (0-10), median (p25 - p75) & - & $3.1(1.3-5.2)$ & - & - \\
\hline HLA-B27, n (\%) & - & $561(76)$ & - & - \\
\hline Erosions (RA), n (\%) & $352(45.4)$ & - & - & - \\
\hline Biologic therapy (\% ever treated), n (\%) & $313(40.4)$ & $349(47.4)$ & $300(41.7)$ & - \\
\hline Vitamin D, n (\%) & $325(41.9)$ & $82(11.1)$ & $114(15.8)$ & $138(20.4)$ \\
\hline Calcium supplements, n (\%) & $328(42.3)$ & $68(9.2)$ & $105(14.6)$ & $122(18.0)$ \\
\hline
\end{tabular}

$25(\mathrm{OH}) \mathrm{D}$ deficiency is defined as $25(\mathrm{OH}) \mathrm{D}<20 \mathrm{ng} / \mathrm{ml}$. *Hours of sunshine per month considering the period of time between 60 and 90 days prior to the baseline visit (blood test to determine the levels of $25(\mathrm{OH}) \mathrm{D}$ was performed at the baseline visit). BMI body mass index, 25(OH)D 25-hydroxyvitamin $\mathrm{D}, p 25-p 7525^{\text {th }}$ to $75^{\text {th }}$ percentile, $R F$ rheumatoid factor, $A C P A$ anti-cyclic citrullinated peptide antibodies, DAS28-ESR, disease activity score using 28 joints-erythrocyte sedimentation rate, $H A Q(0-3)$ : health assessment questionnaire, ESR erythrocyte sedimentation rate, CRP C-reactive protein, BASDAI (0-10) Bath ankylosing spondylitis disease activity index, BASFI (0-10) Bath ankylosing spondylitis functional index, HLA-B27 histocompatibility antigen HLA-B27, RA rheumatoid arthritis

present study raises several points of potential interest. First, the CARMA cohort constitutes the largest series of comparisons of $25(\mathrm{OH}) \mathrm{D}$ levels in three well-established CIRD. In addition, a cohort of individuals without CIRD was used for comparison. Second, we assessed patients who were periodically followed at rheumatology outpatient clinics. Nevertheless, it is important to emphasize that baseline levels of $25(\mathrm{OH}) \mathrm{D}$ in the control population 
Table 2 Bivariate analysis according to each specific entity and the occurrence of $25(\mathrm{OH}) \mathrm{D}$ deficiency $(25(\mathrm{OH}) \mathrm{D}<20 \mathrm{ng} / \mathrm{ml})$

\begin{tabular}{|c|c|c|c|c|c|c|c|c|c|c|c|c|c|c|c|c|}
\hline \multirow[b]{2}{*}{ Variables } & \multicolumn{4}{|l|}{ All } & \multicolumn{3}{|c|}{ Rheumatoid arthritis } & \multicolumn{3}{|c|}{ Ankylosing spondylitis } & \multicolumn{3}{|c|}{ Psoriatic arthritis } & \multicolumn{3}{|l|}{ Controls } \\
\hline & Total & $\begin{array}{l}25 \mathrm{OHD} \\
<20\end{array}$ & $\begin{array}{l}25 \mathrm{OHD} \\
\geq 20\end{array}$ & $p$ & $\begin{array}{l}25 \mathrm{OHD} \\
<20\end{array}$ & $\begin{array}{l}25 \mathrm{OHD} \\
\geq 20\end{array}$ & $p$ & $\begin{array}{l}25 \mathrm{OHD} \\
<20\end{array}$ & $\begin{array}{l}25 \mathrm{OHD} \\
\geq 20\end{array}$ & $p$ & $\begin{array}{l}25 \mathrm{OHD} \\
<20\end{array}$ & $\begin{array}{l}25 \mathrm{OHD} \\
\geq 20\end{array}$ & $p$ & $\begin{array}{l}25 \mathrm{OHD} \\
<20\end{array}$ & $\begin{array}{l}25 \mathrm{OHD} \\
\geq 20\end{array}$ & $p$ \\
\hline Female, n (\%) & $\begin{array}{l}1545 \\
(53.1)\end{array}$ & $554(51.2)$ & 796 (55.0) & 0.054 & $220(70.1)$ & $280(79.5)$ & 0.005 & $92(31.4)$ & $81(23.9)$ & 0.035 & $127(43.1)$ & $162(48.1)$ & 0.206 & $115(63.5)$ & $273(65.2)$ & 0.703 \\
\hline $\begin{array}{l}\text { Age beginning disease, } \\
\text { years, mean (SD) }\end{array}$ & $\begin{array}{l}40.5 \\
(14.5)\end{array}$ & $\begin{array}{l}40.4 \\
(14.5)\end{array}$ & $\begin{array}{l}41.4 \\
(14.7)\end{array}$ & 0.079 & $45.8(13)$ & $\begin{array}{l}46.1 \\
(13.6)\end{array}$ & 0.743 & $30.1(11.7)$ & $\begin{array}{l}29.6 \\
(11.8)\end{array}$ & 0.620 & $\begin{array}{l}39.2 \\
(13.1)\end{array}$ & $\begin{array}{l}39.4 \\
(13.3)\end{array}$ & 0.846 & $49.4(12.6)$ & $\begin{array}{l}48.5 \\
(12.2)\end{array}$ & 0.406 \\
\hline $\begin{array}{l}\text { Disease duration, years, } \\
\text { mean (SD) }\end{array}$ & $\begin{array}{l}11.03 \\
(10.4)\end{array}$ & $\begin{array}{l}11.5 \\
(10.2)\end{array}$ & $\begin{array}{l}10.7 \\
(10.5)\end{array}$ & 0.005 & $10.1(8.5)$ & $10.4(9.1)$ & 0.782 & $17.6(11.9)$ & $\begin{array}{l}17.72 \\
(12.1)\end{array}$ & 0.950 & $11.2(8.4)$ & $11.4(9.3)$ & 0.983 & $4.4(6.9)$ & $4.5(6.2)$ & 0.321 \\
\hline \multicolumn{17}{|l|}{ Educational level, n (\%) } \\
\hline Elementary & $\begin{array}{l}1693 \\
(58.6)\end{array}$ & $646(60.4)$ & $819(56.8)$ & 0.071 & $212(68.6)$ & $242(68.9)$ & 0.926 & $176(60.7)$ & $182(53.8)$ & 0.84 & $173(59.5)$ & $200(59.5)$ & 0.985 & $85(47.5)$ & $195(46.9)$ & 0.891 \\
\hline University/secondary & $\begin{array}{l}1194 \\
(41.4)\end{array}$ & $423(39.6)$ & $622(43.2)$ & & $97(31.4)$ & $109(31.1)$ & & $114(39.3)$ & $156(46.2)$ & & $118(40.5)$ & $136(40.5)$ & & $94(52.5)$ & $221(53.1)$ & \\
\hline \multicolumn{17}{|l|}{ Smoking status, n (\%) } \\
\hline Current smokers & $743(25.5)$ & $303(28.0)$ & $335(23.2)$ & & $83(24.4)$ & $76(21.6)$ & & $107(36.5)$ & $113(33.3)$ & & $59(20.0)$ & 73 (21.6) & & $54(30.0)$ & $73(17.4)$ & \\
\hline Past smokers & $845(29.0)$ & $332(30.7)$ & $407(28.1)$ & 0.001 & $86(27.4)$ & $92(26.1)$ & 0.226 & $95(32.4)$ & $107(31.6)$ & 0.530 & $101(34.2)$ & $104(30.9)$ & 0.650 & $50(27.8)$ & $104(24.8)$ & $<0.001$ \\
\hline Never smokers & $\begin{array}{l}1322 \\
(45.5)\end{array}$ & $447(41.3)$ & $705(48.7)$ & & $145(46.2)$ & $184(52.3)$ & & $91(31.1)$ & $119(35.1)$ & & $135(45.8)$ & $160(47.5)$ & & $76(42.2)$ & $242(57.8)$ & \\
\hline Obesity (BMI $\geq 30), n(\%)$ & $\begin{array}{l}2184 \\
(75.0)\end{array}$ & $327(30.2)$ & $308(21.3)$ & 0.001 & $88(28.0)$ & $71(20.2)$ & 0.018 & $83(28.3)$ & $75(22.1)$ & 0.072 & $102(34.7)$ & $89(26.5)$ & 0.025 & $54(30.0)$ & $73(17.5)$ & 0.001 \\
\hline \multicolumn{17}{|l|}{ Main physical activity, n (\%) } \\
\hline Sedentary & $\begin{array}{l}1043 \\
(39.8)\end{array}$ & $380(39.3)$ & $553(40.8)$ & & $92(34.3)$ & $113(36.1)$ & & $103(39.6)$ & $120(38.7)$ & & $110(41.5)$ & $119(39.1)$ & & $75(42.9)$ & $181(47.6)$ & \\
\hline Moderate & $976(37.2)$ & $363(37.5)$ & $472(36.1)$ & 0.734 & $113(42.2)$ & $136(43.5)$ & 0.669 & $90(34.6)$ & $109(35.2)$ & 0.976 & $100(37.7)$ & $107(35.2)$ & 0.386 & $60(34.3)$ & $120(31.6)$ & 0.576 \\
\hline $\begin{array}{l}\text { Active with } \\
\text { displacement }\end{array}$ & $604(23.0)$ & $225(23.2)$ & $302(23.1)$ & & $63(23.5)$ & $64(20.4)$ & & $67(25.8)$ & $81(26.1)$ & & $55(20.8)$ & $78(25.7)$ & & $40(22.9)$ & 79 (20.8) & \\
\hline $\begin{array}{l}\text { Sunshine hours /month*, } \\
\text { median (p25 - p75) }\end{array}$ & $\begin{array}{l}189 \\
(138-269)\end{array}$ & $\begin{array}{l}161 \\
(122-225)\end{array}$ & $\begin{array}{l}210 \\
(159-320)\end{array}$ & $<0.001$ & $\begin{array}{l}156 \\
(101-193)\end{array}$ & $\begin{array}{l}184 \\
(141-234)\end{array}$ & $<0.001$ & $\begin{array}{l}161 \\
(122-202)\end{array}$ & $\begin{array}{l}200 \\
(157-251)\end{array}$ & $<0.001$ & $\begin{array}{l}159 \\
(103-200)\end{array}$ & $\begin{array}{l}201 \\
(150-263)\end{array}$ & $<0.001$ & $\begin{array}{l}252 \\
(162-345)\end{array}$ & $\begin{array}{l}303 \\
(202-345)\end{array}$ & 0.031 \\
\hline \multicolumn{17}{|l|}{ Clinical characteristics } \\
\hline $\begin{array}{l}\text { ESR }(\mathrm{mm} / \mathrm{h}), \text { median } \\
(\mathrm{p} 25-\mathrm{p} 75)\end{array}$ & - & - & - & - & $\begin{array}{l}18.0 \\
(9.0-28.0)\end{array}$ & $\begin{array}{l}16.0 \\
(9.0-30.0)\end{array}$ & 0.631 & $\begin{array}{l}10.0 \\
(6.0-23.2)\end{array}$ & $\begin{array}{l}11.0 \\
(6.0-19.0)\end{array}$ & 0.691 & $\begin{array}{l}12.0 \\
(5.0-21.0)\end{array}$ & $\begin{array}{l}12.0 \\
(6.0-21.0)\end{array}$ & 0.471 & $\begin{array}{l}11.0 \\
(5.0-18.0)\end{array}$ & $\begin{array}{l}10.0 \\
(5.0-18.0)\end{array}$ & 0.189 \\
\hline $\begin{array}{l}\text { CRP }(\mathrm{mg} / \mathrm{l}), \text { median } \\
(\mathrm{p} 25-\mathrm{p} 75)\end{array}$ & - & - & - & - & $\begin{array}{l}3.1 \\
(1.4-8.0)\end{array}$ & $\begin{array}{l}3.0 \\
(1.0-7.3)\end{array}$ & 0.543 & $\begin{array}{l}4.1 \\
(1.5-10.6)\end{array}$ & $\begin{array}{l}3.2 \\
(1.6-7.6)\end{array}$ & 0.333 & $\begin{array}{l}3.0 \\
(1.4-6.1)\end{array}$ & $\begin{array}{l}2.9 \\
(1.3-6.2)\end{array}$ & 0.449 & $\begin{array}{l}2.0(0.9- \\
4.1)\end{array}$ & $\begin{array}{l}1.9 \\
(1.0-3.2)\end{array}$ & 0.358 \\
\hline RF positive, n (\%) & - & - & - & - & $252(80.3)$ & $264(75.0)$ & 0.105 & - & - & - & - & - & - & - & - & - \\
\hline ACPA positive, $n(\%)$ & - & - & - & - & $210(66.9)$ & $205(58.2)$ & 0.022 & - & - & - & - & - & - & - & - & - \\
\hline Erosions, n (\%) & - & - & - & - & $143(45.5)$ & $169(48.0)$ & 0.524 & - & - & - & 79 (26.8) & $92(27.3)$ & 0.883 & - & - & - \\
\hline $\begin{array}{l}\text { DAS28-ESR, median } \\
\text { (p25 - p75) }\end{array}$ & - & - & - & - & $\begin{array}{l}3.0 \\
(2.2-3.8)\end{array}$ & $\begin{array}{l}3.0 \\
(2.3-4.0)\end{array}$ & 0.937 & - & - & - & $\begin{array}{l}2.8 \\
(1.9-3.9)\end{array}$ & $\begin{array}{l}2.9 \\
(2.0-3.9)\end{array}$ & 0.807 & - & - & - \\
\hline
\end{tabular}


Table 2 Bivariate analysis according to each specific entity and the occurrence of 25(OH)D deficiency $(25(\mathrm{OH}) \mathrm{D}<20 \mathrm{ng} / \mathrm{ml})(\mathrm{Continued})$

\begin{tabular}{|c|c|c|c|c|c|c|c|c|c|c|c|c|c|c|c|c|}
\hline $\begin{array}{l}\text { HAQ (0-3), median } \\
\text { (p25-p75) }\end{array}$ & - & - & - & - & $\begin{array}{l}0.5 \\
(0.1-1.1)\end{array}$ & $\begin{array}{l}0.6 \\
(0.1-1.2)\end{array}$ & 0.193 & - & - & - & $\begin{array}{l}0.2 \\
(0.0-1.0)\end{array}$ & $\begin{array}{l}0.3 \\
(0.0-1.9)\end{array}$ & 0.601 & - & - & - \\
\hline HLA-B27, n (\%) & - & - & - & - & - & - & - & $218(74.4)$ & $275(81.1)$ & 0.042 & & - & - & - & - & - \\
\hline $\begin{array}{l}\text { BASDAI (0-10), median } \\
\text { (p25 - p75) }\end{array}$ & - & - & - & - & - & - & - & $\begin{array}{l}3.8 \\
(1.8-5.3)\end{array}$ & $\begin{array}{l}3.3 \\
(1.8-5.0)\end{array}$ & 0.042 & - & - & - & - & - & - \\
\hline $\begin{array}{l}\text { BASFI (0-10), median } \\
(\mathrm{p} 25-\mathrm{p} 75)\end{array}$ & - & - & - & - & - & - & - & $\begin{array}{l}3.7 \\
(1.6-5.8)\end{array}$ & $\begin{array}{l}2.7 \\
(1.2-4.7)\end{array}$ & 0.018 & - & - & - & - & - & - \\
\hline \multicolumn{17}{|l|}{ Treatment } \\
\hline Biologic DMARD, n (\%) & - & - & - & - & $123(39.2)$ & $150(42.6)$ & 0.367 & $145(49.5)$ & $158(46.6)$ & 0.470 & $132(44.7)$ & $142(42.1)$ & 0.509 & - & - & - \\
\hline Vitamin D, n (\%) & $659(22.6)$ & $198(18.3)$ & $392(27.1)$ & $<0.001$ & $120(38.2)$ & $166(47.2)$ & 0.020 & $23(7.8)$ & $53(15.6)$ & 0.003 & $32(10.8)$ & $68(20.2)$ & 0.001 & $23(12.7)$ & $105(25.1)$ & $<0.001$ \\
\hline $\begin{array}{l}\text { Calcium supplements, } \\
\mathrm{n}(\%)\end{array}$ & $623(21.4)$ & $191(17.6)$ & $353(24.4)$ & $<0.001$ & $118(37.6)$ & $163(46.3)$ & 0.023 & $20(6.8)$ & $42(12.4)$ & 0.019 & $33(11.2)$ & 56 (16.6) & 0.050 & $20(11.0)$ & $92(22.0)$ & 0.002 \\
\hline
\end{tabular}

*Hours of sunshine per month considering the period of time between 60 and 90 days prior to the baseline visit (blood test to determine the levels of $25(\mathrm{OH}) \mathrm{D}$ was performed at the baseline visit). $25(\mathrm{OH}) \mathrm{D} 25-$

hydroxyvitamin D, BMI body mass index, $p 25-p 7525^{\text {th }}$ to $75^{\text {th }}$ percentile, ESR erythrocyte sedimentation rate, CRP C-reactive protein, RF rheumatoid factor, ACPA anti-cyclic citrullinated peptide antibodies, DAS28-ESR, disease activity score using 28 joints-erythrocyte sedimentation rate, HAQ (0-3): health assessment questionnaire, HLA-B27 histocompatibility antigen HLA-B27, BASDAI (0-10) Bath ankylosing spondylitis disease activity disease activity score using 28 joints-erythrocyte sedimentation rate, $H A Q(0-3)$ : health assessment questionnaire,
index, BASFI (0-10) Bath ankylosing spondylitis functional index, DMARD disease-modifying anti-rheumatic drugs 
Table 3 Multivariate analysis of $25(\mathrm{OH}) \mathrm{D}$ deficiency $(25(\mathrm{OH}) \mathrm{D}$ levels $<20 \mathrm{ng} / \mathrm{ml})$ in patients with chronic inflammatory rheumatic diseases

\begin{tabular}{|c|c|c|c|c|c|c|c|c|c|c|c|c|}
\hline \multirow{3}{*}{ Variables } & \multicolumn{3}{|l|}{ All } & \multicolumn{3}{|c|}{ Rheumatoid arthritis } & \multicolumn{3}{|c|}{ Ankylosing spondylitis } & \multicolumn{3}{|l|}{ Psoriatic arthritis } \\
\hline & Crude OR & Adjusted OR & $p$ & Crude OR & Adjusted OR & $p$ & Crude OR & Adjusted OR & $p$ & Crude OR & Adjusted OR & $p$ \\
\hline & $(95 \% \mathrm{Cl})$ & $(95 \% \mathrm{Cl})$ & & $(95 \% \mathrm{Cl})$ & $(95 \% \mathrm{Cl})$ & & $(95 \% \mathrm{Cl})$ & $(95 \% \mathrm{Cl})$ & & $(95 \% \mathrm{Cl})$ & $(95 \% \mathrm{Cl})$ & \\
\hline \multicolumn{13}{|l|}{$\begin{array}{l}\text { Kind of disease (ref. } \\
\text { controls) }\end{array}$} \\
\hline Rheumatoid arthritis & $2.07(1.53,2.79)$ & $1.46(1.09,1.96)$ & 0.012 & - & - & & - & - & & - & - & \\
\hline Ankylosing spondylitis & $2.00(1.46,2.74)$ & $1.23(0.85,1.80)$ & 0.273 & - & - & & - & - & & - & - & \\
\hline Psoriatic arthritis & $2.03(1.46,2.81)$ & $1.32(0.94,1.84)$ & 0.110 & - & - & & - & - & & - & - & \\
\hline Age beginning disease & $1.00(0.99,1.00)$ & $1.00(0.99,1.01)$ & 0.985 & $1.00(0.99,1.01)$ & $0.99(0.98,1.01)$ & 0.324 & $1.00(0.99,1.02)$ & $1.00(0.98,1.02)$ & 0.715 & $1.00(0.99,1.01)$ & $1.00(0.98,1.01)$ & 0.688 \\
\hline Sex (ref. male) & $0.86(0.74,0.99)$ & $1.06(0.88,1.27)$ & 0.561 & $0.60(0.43,0.85)$ & $0.64(0.42,0.97)$ & 0.037 & $1.46(1.07,1.99)$ & $1.54(1.10,2.17)$ & 0.013 & $0.82(0.61,1.10)$ & $0.95(0.66,1.37)$ & 0.781 \\
\hline Disease duration, years & $1.01(1.00,1.02)$ & $1.00(0.99,1.01)$ & 0.894 & $1.00(0.98,1.01)$ & $1.00(0.98,1.02)$ & 0.971 & $1.00(0.98,1.01)$ & $1.00(0.98,1.02)$ & 0.841 & $0.998(0.980,1.015)$ & $1.00(0.98,1.01)$ & 0.581 \\
\hline \multicolumn{13}{|l|}{$\begin{array}{l}\text { Educational level (ref. } \\
\text { elementary) }\end{array}$} \\
\hline University/secondary & $0.86(0.67,1.11)$ & $0.99(0.77,1.29)$ & 0.963 & $1.02(0.71,1.45)$ & $1.17(0.69,1.72)$ & 0.430 & $0.76(0.52,1.10)$ & $0.81(0.55,1.19)$ & 0.280 & $1.00(0.67,1.50)$ & $0.94(0.62,1.41)$ & 0.765 \\
\hline \multicolumn{13}{|l|}{$\begin{array}{l}\text { Smoking status (ref. } \\
\text { current smokers) }\end{array}$} \\
\hline Past smokers & $0.90(0.74,1.10)$ & $0.91(0.73,1.13)$ & 0.387 & $0.86(0.58,1.26)$ & $0.80(0.49,1.29)$ & 0.354 & $0.94(0.62,1.41)$ & $1.11(0.72,1.70)$ & 0.639 & $1.20(0.77,1.88)$ & $1.20(0.72,1.99)$ & 0.480 \\
\hline Never smokers & $0.70(0.57,0.86)$ & $0.73(0.58,0.91)$ & 0.005 & $0.72(0.51,1.02)$ & $0.74(0.50,1.11)$ & 0.144 & $0.81(0.53,1.22)$ & $0.84(0.56,1.26)$ & 0.404 & $1.04(0.66,1.64)$ & $1.12(0.67,1.87)$ & 0.662 \\
\hline Obesity (BMI $\geq 30 . \mathrm{kg} / \mathrm{m}^{2}$ ) & $1.60(1.32,1.94)$ & $1.96(1.28,1.90)$ & $<0.001$ & $1.54(1.09,2.17)$ & $1.76(1.18,2.62)$ & 0.006 & $1.39(0.93,2.09)$ & $1.20(0.78,1.86)$ & 0.408 & $1.47(1.04,2.09)$ & $1.41(0.98,2.05)$ & 0.067 \\
\hline Sunshine hours/month* & $0.99(0.99,0.99)$ & $0.99(0.99,0.99)$ & $<0.001$ & $0.99(0.99,0.99)$ & $0.99(0.99,0.99)$ & 0.001 & $0.99(0.99,0.99)$ & $0.99(0.99,0.99)$ & 0.001 & $0.99(0.99,0.99)$ & $0.99(0.99,0.99)$ & $<0.001$ \\
\hline HAQ (0-3) & & & & $0.86(0.69,1.07)$ & $0.90(0.71,1.14)$ & 0.384 & - & - & & - & - & \\
\hline ACPA-positive & - & - & & $1.45(1.00,2.09)$ & $1.45(0.99,2.12)$ & 0.056 & - & - & & - & - & \\
\hline HLA-B27 & - & - & & - & - & & $0.68(0.50,0.92)$ & $0.70(0.48,1.02)$ & 0.062 & - & - & \\
\hline BASFI (0-10) & - & - & & - & - & & $1.10(1.02,1.18)$ & $1.08(0.99,1.17)$ & 0.070 & - & - & \\
\hline Vitamin D therapy & $0.60(0.47,0.77)$ & $0.57(0.43,0.76)$ & $<0.001$ & $0.69(0.48,0.99)$ & $0.78(0.52,1.18)$ & 0.245 & $0.46(0.26,0.81)$ & $0.43(0.26,0.81)$ & 0.008 & $0.48(0.28,0.82)$ & $0.54(0.29,1.01)$ & 0.053 \\
\hline
\end{tabular}

\section{*Hours of sunshine per month considering the period of time between 60 and 90 days prior to the baseline visit (blood test to determine the levels of 25(OH)D was performed at the baseline visit). 25(OH)D 25-} hydroxyvitamin D, OR odds ratio, BMI body mass index, HAQ (0-3): health assessment questionnaire, ACPA anti-cyclic citrullinated peptide antibodies, HLA-B27 histocompatibility antigen HLA-B27, BASFI (0-10) Bath ankylosing spondylitis functional index 
were also low, due to the inclusion in this population of a high percentage of subjects with osteoarthritis and/or osteoporosis, who are also likely to have low baseline 25 $(\mathrm{OH}) \mathrm{D}$ levels.

Nowadays it is not clear whether vitamin D deficiency is the cause or effect of the inflammatory process. In this regard, in a model of acute phase response after surgery, plasma concentrations of $25(\mathrm{OH}) \mathrm{D}$ were found to decrease after elective knee arthroplasty [32]. Furthermore, several studies have found an inverse association between 25(OH)D levels and activity parameters of some CIRD, such as DAS28, swollen joints and HAQ in RA and BASFI and BASDAI in AS $[17,19,21]$. Although the results from our study do not fully support all these findings, in the multivariate analysis a marginally statistically significant association between 25(OH)D deficiency and ACPA in RA and BASFI in AS was found. It is worth noting that our patients with CIRD had decreased 25(OH)D levels despite the fact that a great majority had low activity at the time of inclusion. Patients with CIRD have less mobility and life outdoors, which would also contribute negatively to maintain adequate levels of vitamin $\mathrm{D}$. Therefore, 25(OH)D deficiency in these patients may be explained by a dual mechanism. On the one hand, chronic diseases can predispose to $25(\mathrm{OH}) \mathrm{D}$ deficiency directly by decreasing synthesis or increasing vitamin D catabolism, and on the other hand, indirectly lowering sunlight exposure in phases of reduced mobility and ability to spend time outdoors in patients with worse functional status.

We feel that our results may be considered of potential interest in daily clinical practice, as our population encompassed individuals periodically followed at rheumatology outpatient clinics, many of whom are controlled under biological treatment.

Although a recent umbrella review of systematic reviews and meta-analyses of observational studies and randomized trials did not demonstrate that supplementation of vitamin $\mathrm{D}$ improves the health of the general population [33], we believe it is important to monitor and supplement vitamin D to patients with CIRD and vitamin $\mathrm{D}$ deficiency, regardless of whether the deficiency of vitamin D may or may not have a pathogenic role, or whether it is merely an epiphenomenon associated with inflammatory disease.

Our study has some limitations. First, a potential limitation of the study was that the non-CIRD subjects were not completely healthy, as a high percentage of individuals included had osteoarthritis, osteoporosis and/or other musculoskeletal diseases, which by themselves are associated with some risk of $25(\mathrm{OH}) \mathrm{D}$ deficiency. Another limitation of this study may be that the control group had more sunshine hours because many controls were recruited in the months of October and November, and several studies indicate that the level of $25(\mathrm{OH}) \mathrm{D}$ is the result of sun exposure in a period of time between 60 to 90 days prior to the 25(OH)D assessment [34], which in our study corresponded with the months of July and August. With respect to the variability of the vitamin D measurement among all participating centers, we performed a mixed model of logistic regression to reduce the variability in the method of assessment of $25(\mathrm{OH}) \mathrm{D}$ levels.

Finally, another limitation is the possible ecological fallacy that we may be committing to impute the average hours of sunshine from one province to every individual. As the CARMA study was designed to determine the causality of cardiovascular mortality in patients with CIRD, information on the length of time during which individual patients were exposed to sunshine was not collected. Therefore, and because sun exposure is a key factor in the blood levels of vitamin $\mathrm{D}$, and patients were not recruited in the same period of the year and in the same geographical area of the country, we decided to collect aggregate information on sunshine hours provided by the SMA as an adjustment variable in the multivariate model.

\section{Conclusions}

In summary, patients with RA followed at rheumatology outpatient clinics have high risk of $25(\mathrm{OH}) \mathrm{D}$ deficiency, in spite of presenting low-to-moderate disease activity due to tight control of the disease. In consequence, we believe that we must monitor the levels of vitamin D at baseline and during follow up, and supplement vitamin $\mathrm{D}$ if any deficiency is detected.

\section{Additional file}

Additional file 1: Table S1. Distribution of the patients and controls according to the geographic area (region) and the month of the year of inclusion in the study. (DOC $70 \mathrm{~kb}$ )

\section{Abbreviations}

25(OH) D: 25-hydroxyvitamin D; ACPA: anti-cyclic citrullinated peptide antibodies; ACR: American College of Rheumatology; AS: ankylosing spondylitis; BASDAI (0-10): Bath ankylosing spondylitis disease activity index; BASFI (0-10): Bath ankylosing spondylitis functional index; BMl: body mass index; CARMA: Cardiovascular in rheumatology project; CIRD: chronic inflammatory rheumatic diseases; CRP: C-reactive protein; DAS28-ESR: Disease activity score including 28 joints-erythrocyte sedimentation rate; HAQ (0-3): Health assessment questionnaire; HLA-B27: histocompatibility leucocyte antigen B27; IQR: interquartile range; OR: odds ratio; $\mathrm{p} 25-\mathrm{p} 75$ : $25^{\text {th }}$ to $75^{\text {th }}$ percentile; PSA: psoriatic arthritis; RA: rheumatoid arthritis; RF: rheumatoid factor SLE, systemic lupus erythematosus; SMA: Spanish Meteorological Agency.

\section{Competing interests}

The authors declare that they have no competing interests.

\section{Authors' contributions}

AUA, MAMM and SC carried out the data analysis and drafted the manuscript. CSP helped interpret the data and improve the manuscript. CG. helped develop the study protocol and the manuscript, and also assisted in data interpretation. $J$ helped design the study protocol, interpret the data, 
strengthen the manuscript and also performed the statistical analysis. FDG helped interpret the data and strengthen the manuscript. MAGG helped design and developed the CARMA project, assisted in data interpretation, and was responsible for the final draft of the manuscript. All authors read and approved the final manuscript.

\section{Acknowledgements}

This publication was aided by members of the Research Unit of the SER. Dedicated to Dr José L Fernández Sueiro who took part in the initial design of this project and passed away in 2012. The authors thank all of the health professionals and patients who generously participated in this study. Furthermore, the authors thank the approval of the study from all participating centers: Complejo Hospitalario A Coruña, A Coruña; Instituto Dexeus, Barcelona; Hospital Universitari Vall d'Hebron, Barcelona; Hospital Infanta Sofía, Madrid; Hospital S. Pedro de Alcántara, Cáceres; Hospital Son Llatzer, Palma de Mallorca; Hospital Univ. de Guadalajara; Hospital Clinic i Provincial, Barcelona; Hospital Clínico Univ. San Carlos, Madrid; Hospital de Barbastro, Huesca; Hospital Univ. de Bellvitge, Barcelona; Hospital Univ. de La Princesa, Madrid; Hospital de Mérida, Badajoz; Hospital General Carlos Haya, Málaga; Hospital General Virgen de la Concha, Zamora; Hospital Virgen de la Salud, Toledo; Hospital del Sureste, Madrid; Hospital Ramón y Cajal, Madrid; Hospital Univ. Miguel Servet, Zaragoza; Hospital Dr. Negrín, Las Palmas de Gran Canaria; Hospital de Cabueñes, Gijón; Hospital Gregorio Marañón, Madrid; Hospital Univ. de Salamanca; Hospital Univ. Marqués de Valdecilla, Santander; Hospital de la Marina Baixa, Alicante; Hospital de San Rafael, Barcelona; Hospital General. Universitario, Valencia; Instituto Poal, Barcelona; Hospital Universitario Puerta de Hierro, Madrid; Hospital Clínico Univ. San Cecilio, Granada; Hospital Santiago Apóstol, Vitoria-Gasteiz; Consorci Sanitari de Terrassa, Terrassa; Hospital de Viladecans, Barcelona; Hospital General de Albacete; Hospital Mutua Terrassa, Terrassa; Hospital Ntra. Sra. de Candelaria Santa Cruz de Tenerife; Hospital Univ. de Canarias, La Laguna, Tenerife; Hospital Univ. de Valme, Sevilla; Instituto Provincial de Rehabilitación, Madrid; Hospital de Cantoblanco, Madrid; Hospital de Jerez de la Frontera, Cádiz; Hospital Obispo Polanco, Teruel; Hospital Infanta Leonor, Madrid; Hospital General de Elda, Alicante; Hospital Los Arcos, Murcia; Hospital Severo Ochoa, Madrid; Hospital Príncipe de Asturias, Madrid; Hospital Univ. 12 de Octubre, Madrid; Hospital Univ. Reina Sofía, Córdoba; Hospital Univ. La Paz, Madrid; Hospital Gutiérrez Ortega, Valdepeñas, Ciudad Real; Hospital Virgen de la Arrixaca, Murcia; Hospital de El Escorial, Madrid; Hospital de Basurto, Bilbao; Hospital Dos de Maig, Barcelona; Hospital del Mar, Barcelona; Hospital Universitario Son Espases, Palma de Mallorca; Hospital de Donostia, Donostia; Hospital de la Santa Creu i Sant Pau, Barcelona; Hospital de Palamós, Gerona; Hospital Comarcal de L'Alt Penedés, Vilafranca del Penedès, Barcelona; Hospital Sierrallana, Torrelavega; Complejo Asistencial de León; Hospital General de Ciudad Real; Hospital General de Móstoles, Madrid; Hospital General Universitario de Elche, Alicante; Hospital Xeral Calde, Lugo. This project has been supported by an unrestricted grant from Abbvie, Spain. The design, analysis, interpretation of results and preparation of the manuscript has been done independently of Abbvie. Dr González-Gay's studies have been supported by grants from Fondo de Investigaciones Sanitarias, PI06/0024, PS09/00748 and PI12/00060, and RD12/0009/0013 (RIER) from Instituto de Salud Carlos III (ISCIII) (Spain).

The members of the CARMA Project Collaborative Group include: José L Fernández Sueiro and Eugenia Gonzalez de Rábago (Complejo Hospitalario A Coruña, Xubias de Arriba, A Coruña); María J. González Fernández, Ramón Huguet Codina, Beatriz Yoldi and Mercedes Ramentol (Instituto Dexeus, Barcelona); Sara Marsal, Gabriela Ávila and Cayetano Alegre (Hospital Universitari Vall d'Hebron, Barcelona); Martina Steiner, Tatiana Cobo and Santiago Muñoz (Hospital Infanta Sofía, Madrid); Fernando Gamero, José García Torón and María P. Moreno Gil (Hospital S. Pedro de Alcántara, Cáceres); Antonio J. Mas, Pilar Espiño, Inmaculada Ros and Mónica Ibañez (Hospital Son Llatzer, Palma de Mallorca); Jesús Tornero and José A. Piqueras (Hospital Univ. de Guadalajara); Raimon Sanmartí and Horacio Berman (Hospital Clinic i Provincial, Barcelona); Oscar Fontseré Patón, Benjamín Fernández Gutiérrez and Lydia Abasolo (Hospital Clínico Univ. San Carlos, Madrid); José M. Pina Salvador and María D. Fábregas (Hospital de Barbastro, Huesca); Montserrat Romera and Joan M. Nolla (Hospital Univ. de Bellvitge, Barcelona); Miriam García Arias, Jesús A. García Vadillo and Rosario García de Vicuña (Hospital Univ. de La Princesa, Madrid); Eugenio Chamizo Carmona (Hospital de Mérida, Badajoz); Antonio Fernández. Nebro, Inmaculada Ureña, María A. Belmonte and María V. Irigoyen (Hospital General Carlos Haya, Málaga); Olga Martínez
González (Hospital General Virgen de la Concha); Rebeca Belmonte Gómez, Pastora Granados Bautista, Azucena Hernández Sanz and José Santos Rey (Hospital Virgen de la Salud, Toledo); Carmen O. Sánchez González (Hospital del Sureste, Madrid); Javier Bachiller and Antonio Zea (Hospital Ramón y Cajal, Madrid); Francisco J. Manero, Chesús Beltrán Audera, Marta Medrano and Ángela Pecondón (Hospital Univ. Miguel Servet, Zaragoza); Celia Erausquin, Soledad Ojeda and Carlos Rodríguez. Lozano (Hospital Dr. Negrín, Las Palmas de Gran Canaria); Jesús Babío Herráez (Hospital de Cabueñes, Gijón); Francisco J. López Longo, Luis Carreño and Indalecio Monteagudo (Hospital Gregorio Marañón, Madrid); Javier del Pino and Ruth López González (Hospital Univ. de Salamanca); Miguel A. González-Gay, Alfonso Corrales and María Enriqueta Peiró (Hospital Univ. Marqués de Valdecilla, Santander); José M. Senabre and José C. Rosas (Hospital de la Marina Baixa, Alicante); Isabel Rotés, Estefanía Moreno and Alba Erra (Hospital de San Rafael, Barcelona); Javier Calvo and Amalia Rueda (Hospital General. Universitario, Valencia); Ingrid Möller and Isabel Rodríguez (Instituto Poal, Barcelona); Carmen Barbadillo (Hospital Universitario Puerta de Hierro, Madrid); Enrique Raya, Pilar Morales and Ana Nieto (Hospital Clínico Univ. San Cecilio, Granada); Ana Ruibal Escribano (Hospital Santiago Apóstol, Vitoria-Gasteiz); Carmen García Gómez (Consorci Sanitari de Terrassa, Terrassa); Sergio Ros Expósito (Hospital de Viladecans, Barcelona); Ginés Sánchez Nievas, Enrique Júdez Navarro and Manuela Sianes Fernández (Hospital General de Albacete); Silvia Martínez. Pardo and Manel Pujol (Hospital Mutua Terrassa, Terrassa); Beatriz Gónzález Alvarez and Alberto Cantabrana (Hospital Ntra. Sra. de Candelaria, Santa Cruz de Tenerife); Sagrario Bustabad and Esmeralda Delgada (Hospital Univ. de Canarias, La Laguna, Tenerife); Alejandro Muñoz and Sergio Rodríguez Montero (Hospital Univ. de Valme, Sevilla); Javier Rivera Redondo and Teresa González Hernández (Instituto Provincial de Rehabilitación, Madrid); Francisco J. González. Polo (Hospital de Cantoblanco, Madrid); Raúl Menor Almagro (Hospital de Jerez de la Frontera, Jerez de la Frontera, Cádiz); José M. Moreno and Emilio Giner Serret (Hospital Obispo Polanco, Teruel); Laura Cebrián Méndez and María Teresa Navío (Hospital Infanta Leonor, Madrid); Cristina Fernández Carballido (Hospital General de Elda, Alicante); Encarnación Pagán and Pablo Mesa del Castillo (Hospital Los Arcos, Murcia); Esperanza Naredo and Ana Cruz (Hospital Severo Ochoa, Madrid); Ana Turrión (Hospital Príncipe de Asturias, Madrid); Isabel Mateo, Julio Sánchez and María Galindo (Hospital Univ. 12 de Octubre, Madrid); Eduardo Collantes, Desireé Ruíz and Pilar Font (Hospital Univ. Reina Sofía, Córdoba); Gema Bonilla (Hospital Univ. La Paz, Madrid); Antonio López Meseguer (Hospital Gutiérrez Ortega, Valdepeñas, Ciudad Real); Manuel J. Moreno and Luis F. Linares (Hospital Virgen de la Arrixaca, Murcia); Mercedes Morcillo and María L. González Gómez (Hospital del Escorial, Madrid); María L. García Vivar, Natalia A. Rivera and Olaia Fernández. Berrizbeitia (Hospital de Basurto, Bilbao); Manel Riera and Yolanda María León (Hospital Dos de Maig, Barcelona); Joan Maymó and Miriam Amirall (Hospital del Mar, Barcelona); Jordi Fiter, Julia Fernández Melón and Luis Espadaler (Hospital Universitario Son Espases, Palma de Mallorca); Joaquín Belzunegui and Inmaculada Bañegil (Hospital de Donostia, Donostia); César Díaz (Hospital de la Santa Creu i Sant Pau, Barcelona); Ramón Valls (Hospital de Palamós, Gerona); Iván Castellví and María Bonet (Hospital Comarcal de L'Alt Penedés, Vilafranca del Penedès, Barcelona); Jaime Calvo Alen (Hospital Sierrallana, Torrelavega); Trinidad Pérez Sandoval (Complejo Asistencial de León); Eva Revuelta Evrard (Hospital General de Ciudad Real); Javier R. Godo (Hospital General de Móstoles, Madrid); Francisco Navarro Blasco (Hospital General Universitario de Elche, Alicante); José A. Miranda-Filloy (Hospital Xeral Calde, Lugo).

\section{Author details}

${ }^{1}$ Division of Rheumatology, Hospital Can Misses, Calle Corona s/n, 07800 Ibiza, Spain. ${ }^{2}$ Research Unit of Spanish Society of Rheumatology, Calle Marqués del Duero, $51^{\circ} \mathrm{A}, 28001$ Madrid, Spain. ${ }^{3}$ Division of Rheumatology, Hospital U de la Princesa, IIS-IPrincesa, Calle Diego de León 62, 28006 Madrid, Spain. ${ }^{4}$ Division of Cardiology, Hospital Lucus Augusti, Lugo 28001, Spain. ${ }^{5}$ Division of Epidemiology and Computational Biology, School of Medicine, University of Cantabria, CIBER Epidemiología y Salud Pública (CIBERESP), Santander, Spain. ${ }^{6}$ Research Unit of Spanish Society of Rheumatology, Madrid, Spain. ${ }^{7}$ School of Medicine, Universidad de La Laguna, Tenerife, Spain. ${ }^{8}$ Division of Rheumatology, Hospital Universitario de Canarias, La Laguna, Tenerife, Spain. ${ }^{9}$ Division of Rheumatology, Hospital Universitario Marqués de Valdecilla, Santander, and Epidemiology, Genetics and Atherosclerosis Research Group on Systemic Inflammatory Diseases, Rheumatology Division, IDIVAL, Avenida de Valdecilla, s/n, 39008 Santander, Spain. 
Received: 11 January 2015 Accepted: 2 July 2015

Published online: 14 August 2015

\section{References}

1. Bikle DD. What is new in vitamin D: 2006-2007. Curr Opin Rheumatol. 2007;19:383-8

2. Hewison M. Vitamin D, and the immune system: new perspectives on an old theme. Endocrinol Metab Clin North Am. 2010;39:365-79.

3. Verstuyf A, Carmeliet G, Bouillon R, Mathieu C. Vitamin D: a pleiotropic hormone. Kidney Int. 2010;78:140-5.

4. Arnson Y, Amital H, Shoenfeld Y. Vitamin D and autoimmunity: new aetiological and therapeutic considerations. Ann Rheum Dis. 2007;66:1137-42.

5. Gatenby P, Lucas R, Swaminathan A. Vitamin D deficiency and risk for rheumatic diseases: an update. Curr Opin Rheumatol. 2013;25:184-91.

6. Wobke TK, Sorg BL, Steinhilber D. Vitamin D in inflammatory diseases. Front Physiol. 2014;5:244

7. Brance ML, Brun LR, Lioi S, Sanchez A, Abdala M, Oliveri B. Vitamin D levels and bone mass in rheumatoid arthritis. Rheumatol Int. 2015;35:499-505.

8. Ranganathan P, Khalatbari S, Yalavarthi S, Marder W, Brook R, Kaplan MJ. Vitamin D deficiency, interleukin 17, and vascular function in rheumatoid arthritis. J Rheumatol. 2013;40:1529-34.

9. Souberbielle JC, Body JJ, Lappe JM, Plebani M, Shoenfeld Y, Wang TJ, et al. Vitamin D and musculoskeletal health, cardiovascular disease, autoimmunity and cancer: Recommendations for clinical practice. Autoimmun Rev. 2010;9:709-15.

10. Holick MF. Vitamin D, deficiency. N Engl J Med. 2007:357:266-81.

11. Cutolo M. Vitamin D, or hormone D deficiency in autoimmune rheumatic diseases, including undifferentiated connective tissue disease. Arthritis Res Ther. 2008;10:123.

12. Borba VZ, Vieira JG, Kasamatsu T, Radominski SC, Sato El, Lazaretti-Castro M. Vitamin D deficiency in patients with active systemic lupus erythematosus. Osteoporos Int. 2009;20:427-33

13. Avina-Zubieta JA, Thomas J, Sadatsafavi M, Lehman AJ, Lacaille D. Risk of incident cardiovascular events in patients with rheumatoid arthritis: a meta-analysis of observational studies. Ann Rheum Dis. 2012;71:1524-9.

14. Oelzner P, Muller A, Deschner F, Huller M, Abendroth K, Hein G, et al. Relationship between disease activity and serum levels of vitamin $D$ metabolites and PTH in rheumatoid arthritis. Calcif Tissue Int. 1998;62:193-8.

15. Craig SM, Yu F, Curtis JR, Alarcon GS, Conn DL, Jonas B, et al. Vitamin D status and its associations with disease activity and severity in African Americans with recent-onset rheumatoid arthritis. J Rheumatol. 2010;37:275-81.

16. Cooles FA, Pratt AG, Wilson G, Isaacs JD, Ng WF. Prevalence and diagnostic outcome relating to vitamin $D$ deficiency in new patients presenting to an early arthritis clinic over 12 months. Clin Rheumatol. 2011;30:1137-8.

17. Cutolo M, Otsa K, Laas K, Yprus M, Lehtme R, Secchi ME, et al. Circannual vitamin $d$ serum levels and disease activity in rheumatoid arthritis: Northern versus Southern Europe. Clin Exp Rheumatol. 2006;24:702-4.

18. Haque UJ, Bartlett SJ. Relationships among vitamin D, disease activity, pain and disability in rheumatoid arthritis. Clin Exp Rheumatol. 2010;28:745-7.

19. Patel S, Farragher T, Berry J, Bunn D, Silman A, Symmons D. Association between serum vitamin $D$ metabolite levels and disease activity in patients with early inflammatory polyarthritis. Arthritis Rheum. 2007;56:2143-9.

20. Rossini M, Maddali Bongi S, La Montagna G, Minisola G, Malavolta N, Bernini L, et al. Vitamin $D$ deficiency in rheumatoid arthritis: prevalence, determinants and associations with disease activity and disability. Arthritis Res Ther. 2010;12:R216

21. Zhao S, Duffield SJ, Moots RJ, Goodson NJ. Systematic review of association between vitamin $D$ levels and susceptibility and disease activity of ankylosing spondylitis. Rheumatology (Oxford). 2014;53:1595-603.

22. Erten S, Kucuksahin O, Sahin A, Altunoglu A, Akyol M, Koca C. Decreased plasma vitamin $\mathrm{D}$ levels in patients with undifferentiated spondyloarthritis and ankylosing spondylitis. Intern Med. 2013;52:339-44.

23. Franck H, Keck E. Serum osteocalcin and vitamin D metabolites in patients with ankylosing spondylitis. Ann Rheum Dis. 1993;52:343-6.

24. Touma Z, Eder L, Zisman D, Feld J, Chandran V, Rosen CF, et al. Seasonal variation in vitamin $\mathrm{D}$ levels in psoriatic arthritis patients from different latitudes and its association with clinical outcomes. Arthritis Care Res. 2011:63:1440-7.

25. Castañeda S, Martín-Martínez MA, González-Juanatey C, Llorca J, García-Yébenes MJ, Pérez-Vicente S, et al. Cardiovascular morbidity and associated risk factors in Spanish patients with chronic inflammatory rheumatic diseases attending rheumatology clinics: Baseline data of the CARMA Project. Semin Arthritis Rheum. 2015;44:618-26. doi:10.1016/j. semarthrit.2014.12.002.

26. Arnett FC, Edworthy SM, Bloch DA, McShane DJ, Fries JF, Cooper NS, et al. The American Rheumatism Association 1987 revised criteria for the classification of rheumatoid arthritis. Arthritis Rheum. 1988;31:315-24.

27. Van der Linden S, Valkenburg HA, Cats A. Evaluation of diagnostic criteria for ankylosing spondylitis. A proposal for modification of the New York criteria. Arthritis Rheum. 1984;27:361-8.

28. Moll JM, Wright V. Psoriatic arthritis. Semin Arthritis Rheum. 1973;3:55-78

29. Instituto Nacional de Estadística. IneBase. Número de horas de sol por región estación, años y meses. Madrid: http://www.ine.es/jaxi/tabla.do?path=/t43/ a012/a1998/10/\&file=t200111 b.px\&type=pcaxis. Accessed 13 March 2015.

30. Ruiz-Irastorza G, Egurbide MV, Olivares N, Martinez-Berriotxoa A, Aguirre C. Vitamin $D$ deficiency in systemic lupus erythematosus: prevalence, predictors and clinical consequences. Rheumatology (Oxford). 2008;47:920-3.

31. Obermayer-Pietsch BM, Lange U, Tauber G, Fruhauf G, Fahrleitner A, Dobnig H, et al. Vitamin $\mathrm{D}$ receptor initiation codon polymorphism, bone density and inflammatory activity of patients with ankylosing spondylitis. Osteoporos Int. 2003;14:995-1000.

32. Reid D, Toole BJ, Knox S, Talwar D, Harten J, O'Reilly DS, et al. The relation between acute changes in the systemic inflammatory response and plasma 25-hydroxyvitamin D concentrations after elective knee arthroplasty. Am J Clin Nutr. 2011:93:1006-11.

33. Theodoratou E, Tzoulaki I, Zgaga L, loannidis JP. Vitamin D and multiple health outcomes: umbrella review of systematic reviews and meta-analyses of observational studies and randomised trials. BMJ. 2014;348:92035.

34. Holick MF. McCollum Award Lecture, 1994: Vitamin D - new horizons for the 21st century. Am J Clin Nutr. 1994;60:619-30.

\section{Submit your next manuscript to BioMed Central and take full advantage of:}

- Convenient online submission

- Thorough peer review

- No space constraints or color figure charges

- Immediate publication on acceptance

- Inclusion in PubMed, CAS, Scopus and Google Scholar

- Research which is freely available for redistribution

Submit your manuscript at www.biomedcentral.com/submit 